University of Nebraska - Lincoln

DigitalCommons@University of Nebraska - Lincoln

February 2003

\title{
Dissociative electron attachment near threshold, thermal attachment rates, and vertical attachment energies of chloroalkanes
}

Gordon A. Gallup

University of Nebraska-Lincoln, ggallup1@unl.edu

Kayvan Aflatooni

Fort Hays State University, kaflatoo@fhsu.edu

Paul Burrow

University of Nebraska-Lincoln, pburrow1@unl.edu

Follow this and additional works at: https://digitalcommons.unl.edu/physicsburrow

Part of the Physics Commons

Gallup, Gordon A.; Aflatooni, Kayvan; and Burrow, Paul, "Dissociative electron attachment near threshold, thermal attachment rates, and vertical attachment energies of chloroalkanes" (2003). Paul Burrow Publications. 5.

https://digitalcommons.unl.edu/physicsburrow/5

This Article is brought to you for free and open access by the Research Papers in Physics and Astronomy at DigitalCommons@University of Nebraska - Lincoln. It has been accepted for inclusion in Paul Burrow Publications by an authorized administrator of DigitalCommons@University of Nebraska - Lincoln. 


\title{
Dissociative electron attachment near threshold, thermal attachment rates, and vertical attachment energies of chloroalkanes
}

\author{
G. A. Gallup, K. Aflatooni, ${ }^{\text {a) }}$ and P. D. Burrow ${ }^{\text {b) }}$ \\ Department of Physics and Astronomy, University of Nebraska-Lincoln, Lincoln, Nebraska 68588-0111
}

(Received 3 September 2002; accepted 15 November 2002)

\begin{abstract}
The peaks appearing near zero energy in the dissociative electron attachment cross section of 18 chloroalkanes are studied by electron beam methods. Fits to the experimental data are made using model cross sections having appropriate energy dependences and inclusion of the broadening due to the electron energy distribution. The magnitudes of the zero peaks are found to be well correlated with the vertical attachment energies (VAE) associated with occupation of the lowest empty orbitals of the compounds. The magnitudes rise exponentially by more than five orders of magnitude as VAE decreases from $2 \mathrm{eV}$ to a slightly negative value. This dependence is a consequence not only of the thermal population of vibrational levels, but also of an approximately linear relationship between VAE and the energy of the crossing between the neutral and anion potential curves. Franck-Condon factors for the transition to the anion curve are computed for model potential curves, and the nature of the attachment from vibrational levels with energies near that of the crossing point is explored in a local potential resonance picture. A substantial contribution arises from tunneling to the anion state from vibrational levels below the barrier. Thermal attachment rate constants are also computed from our data. These are also shown to vary exponentially with VAE. (C) 2003 American Institute of Physics. [DOI: 10.1063/1.1535891]
\end{abstract}

\section{INTRODUCTION}

In work described in detail elsewhere, ${ }^{1-4}$ we have measured the total dissociative electron attachment (DEA) cross sections of a series of polychloroalkanes and chlorofluoromethanes using electron beam techniques. Figure 1 presents an example of these data, showing the DEA cross section of 1,3-dichloropropane as a function of electron impact energy. The line at $1.91 \mathrm{eV}$ locates the lowest vertical attachment energy (VAE) of this compound as determined by electron transmission spectroscopy $(\mathrm{ETS})^{5}$ in a separate experiment. ${ }^{2}$ In the equilibrium geometry of the neutral molecule, the most probable electron attachment into the lowest unoccupied molecular orbital (LUMO) occurs at this energy. In the chloroalkanes this orbital can be represented as a linear combination of one or more of the antibonding $\mathrm{C}-\mathrm{Cl} \sigma^{*}$ local orbitals. The bell-shaped DEA curve peaking at $1.14 \mathrm{eV}$ reflects the energy dependence of the decay of this anion state into the dissociative channel, producing $\mathrm{Cl}^{-}$fragments. The shift in energy between VAE and the energy of the peak in the DEA cross section is a manifestation of the short lifetime of the temporary anion state. ${ }^{6}$

The primary outcome of our earlier work ${ }^{1,3,4}$ was to show that the peak DEA cross sections in these families of molecules are strongly correlated with the vertical attachment energies (VAEs). Furthermore, from ETS measurements of the spread in energy of the temporary anion resonances as they appear in the total electron scattering cross sections and the variation of these widths with VAE, we

${ }^{a)}$ Present address: Department of Physics, Fort Hays State University, Hays, KS 67601.

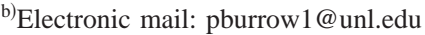

infer $^{2}$ that the lifetimes for autodetachment of the chloroalkane temporary anion states are characteristic of electron tunneling through an $\ell=1$ angular momentum barrier. This result is consistent with the $p_{\sigma}$ character of the $\mathrm{C}-\mathrm{Cl} \sigma^{*}$ orbitals in the LUMOs, and in the remainder of this article we will, for brevity, refer to these features as the " $p$ wave" peaks in the DEA cross sections.

Figure 1 also shows an additional feature common to all our data in the chloroalkanes, namely, a narrow peak at nominally zero electron energy. Although such peaks, to be referred to here as "zero energy" peaks, could arise in principle from additional low-lying temporary anion valence states missed by our ETS measurements, the empty orbital structure of these compounds is well understood, ${ }^{2,7}$ and this possibility can be discounted.

From studies of the temperature dependence of the electron attachment process in similar molecules, the existence of an energy of activation was inferred, ${ }^{8}$ and numerous studies over the years have shown that increasing the internal energy of such molecules causes the DEA cross section at low energies to be greatly enhanced. Thus, it is generally agreed that the zero peaks signal the attachment of electrons to vibrationally excited levels of the neutral molecules lying near the activation energy determined by the crossing point between the neutral potential curve and that of the anion.

In our previous work, the good correlation between the maxima in the $p$ wave DEA cross sections and the VAEs of the chloroalkanes provided evidence that the properties of the temporary anion states associated with electron occupation of the LUMOs are relatively well behaved over this family of molecules. That is, of the $\approx 40$ compounds studied, only one $\left(\mathrm{CH}_{2} \mathrm{Cl}_{2}\right)$ departs significantly from the best-fit cor- 


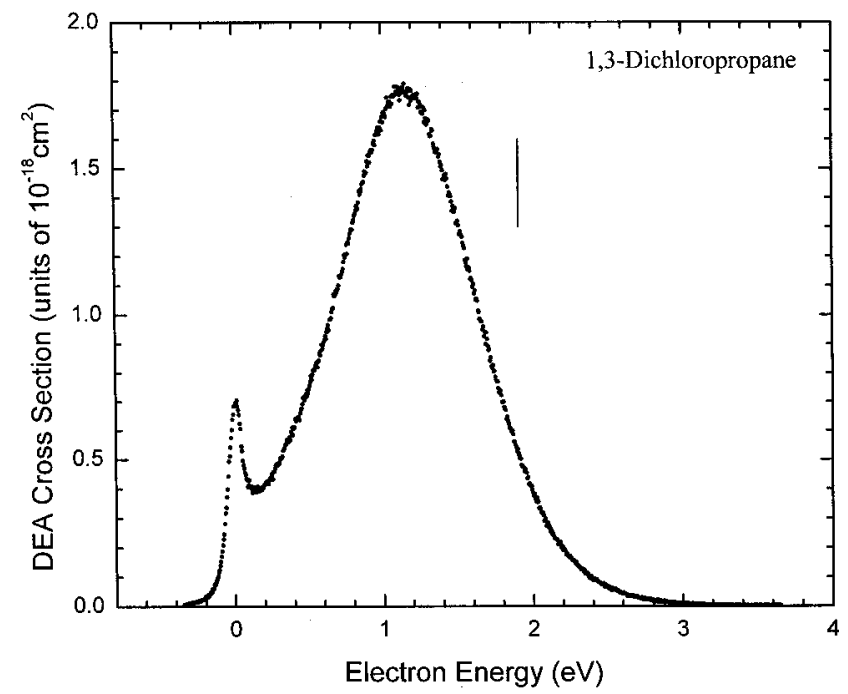

FIG. 1. The cross section for dissociative electron attachment of 1,3dichloropropane as a function of electron impact energy. The vertical line indicates the energy for vertical electron attachment into the lowest unoccupied molecular orbital. The data are from Ref. 1.

relation line. ${ }^{1}$ The average deviation of the cross sections of the remaining compounds from the line is $38 \%$.

In the present work we explore the zero energy peaks measured at room temperature in 18 of the chloroalkanes to determine the degree to which their magnitudes may also be correlated with those properties of the temporary anions responsible for the $p$ wave peaks in the DEA cross sections. The existence of such trends will give insight into the relative positions of the anion and neutral potential curves and also permit us to make predictions about other members of the chloroalkane family. We also calculate the FranckCondon factors for the electron attachment process and examine the extent to which heavy particle tunneling to the anion curve takes place. Finally, we utilize our measured cross sections to compute thermal electron attachment rate constants for these molecules and show that this parameter is also correlated with VAE. Rate constants obtained by other techniques are available for seven of the chloroalkanes in our study, and we compare these with the corresponding values calculated from our zero energy peaks.

\section{DISCUSSION}

\section{A. Fitting the zero energy peaks}

To obtain a more quantitative measure of the magnitude of the zero energy peaks and to aid in separating them from the rising cross sections at higher energy, it is useful to fit the DEA cross sections at low energies with analytic expressions. The natural widths of the peaks are certainly less than that of our electron energy distribution, and thus the magnitudes as they appear in our data are determined by the convolution of the actual cross section with our energy distribution.

The details of the threshold electron energy dependence of the attachment process are closely tied to the target molecular structure, ${ }^{9}$ and even a cursory examination of the beam and swarm literature yields differing interpretations of this fundamentally important aspect of the scattering event. In some cases, for example, it is attributed to an $E^{-1}$ dependence arising from the theoretical maximum inelastic (or reaction) cross section for the $s$ wave, namely $\pi \chi^{2}$, where $\lambda$ is the de Broglie wavelength of the electron. However, as Fabrikant and Hotop ${ }^{9}$ have emphasized in a discussion of threshold DEA effects, the Bethe-Wigner threshold law ${ }^{10,11}$ for nonpolar molecules indicates that the cross section should depend on energy as $E^{\ell-1 / 2}$, where $\ell$ is the lowest allowed angular momentum quantum number. For example, in $\mathrm{CCl}_{4}, \ell=0$, and Klar et al. ${ }^{12}$ have shown with a high resolution measurement that the dissociative electron attachment process for this molecule approaches an $E^{-1 / 2}$ dependence at the lowest energies accessible. Additional complications ensue in polar molecules, as discussed below.

In early studies Bardsley et al. ${ }^{13}$ and $\mathrm{O}^{\prime} \mathrm{Malley}^{6}$ arrived at the expression

$$
\sigma_{\mathrm{DEA}}=\frac{\pi^{2}}{E} \Gamma\left|F_{C}\right|^{2} s
$$

for the DEA cross section, where $E$ is the electron energy, $\Gamma$ is the width of the temporary anion state (proportional to its inverse lifetime) to which the DEA is attributed, $\left|F_{C}\right|^{2}$ is the Franck-Condon factor for the attachment transition between the neutral molecule plus free electron and the anion state, and $s$ is the survival factor. Equation (1a) was derived assuming a local potential for the nuclear motion. As pointed out by Levine (see O'Malley ${ }^{14}$ ) and Domcke, ${ }^{15}$ the theory of DEA should, strictly speaking, be formulated using a nonlocal potential. Nevertheless, a model based upon a local potential will serve our purposes and is more transparent physically. Thus, we will assume that the zero energy peaks can be described by an expression of the effective range sort (in the sense of an expansion in powers of $E$ ), where at least some of the terms may arise from higher energy resonance phenomena in the molecule, and we use a general expression appropriate at very low electron energies

$$
\sigma_{\mathrm{DEA}}=E^{\lambda-1 / 2} C(E)\left|F_{C}\right|^{2} .
$$

In Eq. (1b), $C(E)$ contains the effects of any resonances as well as other contributions to the cross section. For our use at very low energies we will assume that the survival factor is close to 1 , so we omit it here. We discuss the general nature of $\lambda$ and $C(E)$ in Appendix $\mathrm{A}$, where we show that $\lambda$ is expected to vary between 0 and $-1 / 2$ for our molecules at low energy and $C(E)$ is finite at $E=0$. The cross section should therefore behave as $E^{\lambda-1 / 2}$.

Our previous studies ${ }^{1,2}$ show that the widths of the temporary anion states, as they appear in the total scattering cross sections of the chloroalkanes, are consistent with $p$ wave behavior as judged by their dependence on VAE. The symmetries of these molecules, however, do not preclude other partial waves, including the $s$ wave, from the description of the anionic wave function. As $E \rightarrow 0$, we expect the contribution from the $p$ wave, and higher components, to go to zero, leaving only that from the $s$ wave. Thus, we choose a simple additive approximation to the DEA cross section at low energies given by 


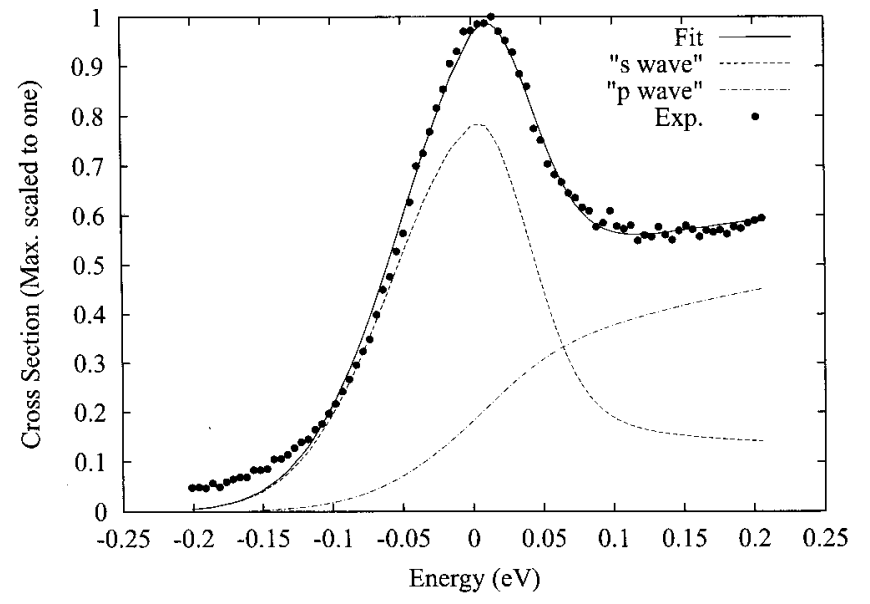

FIG. 2. The relative dissociative electron attachment cross section of 1,3dichloropropane near threshold. The solid line shows the overall fit to the data. The dashed and chain lines indicate the $s$ and $p$ wave contributions to the cross section, respectively, convoluted with the electron energy distribution.

$$
\sigma_{\mathrm{DEA}}(E)=A_{1} E^{a}+A_{2} E^{(a+1)}+B_{1} E^{b} .
$$

The first two terms in Eq. (2) consist of an effective range series expansion for the divergent portion of the cross section as $E \rightarrow 0$. The third term accounts for the rising portion as $E$ increases. Three other adjustable parameters are employed to describe the electron beam distribution, namely $\Delta E_{o}$, a small energy shift in the electron energy scale, an instrument energy width function, $\Gamma_{i}$, representing the full-width at half maximum of the distribution, and an instrument function asymmetry, $A_{i}$. These quantities are related by $\Gamma_{\text {low }}=A_{i} \Gamma_{i}$ and $\Gamma_{\text {high }}=\left(1-A_{i}\right) \Gamma_{i}$. The two half functions are taken to be Gaussians, and $\Gamma_{\text {low }}$ and $\Gamma_{\text {high }}$ characterize the half-widths of the low and high portions of the electron energy distribution, respectively. The latter are required because tuning of a trochoidal electron monochromator ${ }^{16}$ in our experience often produces an asymmetrical energy distribution. A simplex minimization was carried out to determine the fitting parameters.

Figure 2 displays the quality of the analytic fit to DEA data in 1,3-dichloropropane over the energy range $\pm 0.2 \mathrm{eV}$ around nominal zero energy. The experimental points are shown with filled circles and the total fit with a continuous line. The contribution from the first two terms of Eq. (2), the nominally $s$ wave portion, is shown with a dashed line, and that from the third term, the $p$ wave portion, by a chain line. Similar fits were carried out in a total of 18 chloroalkanes, comprising 12 dichloro-, 5 trichloroalkanes, and $\mathrm{CCl}_{4}$. Monochloroalkanes, with generally smaller cross sections, were not included because the data at zero energy are more susceptible to contamination by trace amounts of impurities such as $\mathrm{CCl}_{4} \cdot{ }^{17}$ The figures in Appendix B display fitting results for several of the remaining compounds, along with tables summarizing the fitting parameters.

\section{B. Comments on the fitting results}

Our primary concern is to separate the zero energy peaks from the remaining contributions to the cross sections. However, a few comments on the parameters determined by the fitting process are in order. In the absence of static electric dipole moments, we would expect the energy exponents in Eq. (2) to be $a=-1 / 2$ and $b=+1 / 2$, corresponding to $s$ and $p$ wave behavior, respectively. In the presence of molecular dipole moments, the situation is much more complex, ${ }^{9,18}$ with angular momentum quantum numbers, and consequently the energy exponents, altered by amounts depending on the magnitude of the dipole moments. Thus, parameter $a$ may span a range from $-1 / 2$, in a molecule with zero moment, to -1 in molecules with moments above the critical value (1.62D). Even this is oversimplified, since at very low electron impact energies, $a$ must revert to $-1 / 2$ even in molecules with supercritical dipole moments because of rotational "averaging out" of the moment. Parameter $b$, indicating the nominally $p$ wave dependence, is also dipole moment dependent but more weakly, increasing slightly from $+1 / 2$ with increasing moment. ${ }^{18}$

Although parameters $a$ and $b$, as shown in Appendix B, are consistent with this behavior in an average sense over the family of molecules, clearly our simple fitting approximations do not contain all the physics near the threshold region, nor is our electron beam resolution adequate for this task. In the absence of detailed knowledge of the conformers of each of the compounds and their associated dipole moments, we defer attempts to interpret these parameters more closely to future work. As is evident in Fig. 2 and the figures in Appendix $\mathrm{B}$, the fits to the experimental cross sections are quite acceptable in almost all the compounds and will suffice for the applications in this paper.

\section{Integrated zero peak cross sections}

Having fit the near-threshold DEA cross sections to analytic functions, we now employ them to derive a quantity proportional to the peaked contribution to the cross section near zero energy. As a quantitative measure, we have chosen, somewhat arbitrarily, to integrate the peaked functions from $-0.2 \mathrm{eV}$ to the energy of the maximum in the peak. Tests with an upper integration limit at $0.05 \mathrm{eV}$, thus encompassing most of the peak, show that the trends in our final results are not sensitive to this choice. Table I summarizes the energy integrated cross sections for the half-peak and the VAEs. ${ }^{2}$ The 18 compounds are numbered and listed in order of increasing VAE. The absolute magnitudes of the zero peaks are determined by reference to the measured DEA cross sections of the $p$ wave peaks. ${ }^{1}$

The procedure for $\mathrm{CCl}_{4}$ was somewhat different. Electron attachment data for $\mathrm{CCl}_{4}$ are taken from the earlier work of $\mathrm{Chu}$ and Burrow ${ }^{17}$ but normalized as described in Ref. 1. As discussed elsewhere, ${ }^{19}$ the VAE associated with adding an electron into the LUMO of $\mathrm{CCl}_{4}$ is negative, that is, the ${ }^{2} A_{1}$ ground state of the anion is vertically stable and thus inaccessible by ETS. There is no consensus for the VAE of this state. Koopmans' theorem calculations incorporating the stabilization method were carried out by Falcetta and Jordan ${ }^{7}$ in the chloromethanes. All of the calculated VAEs were shifted by the fixed amount required to match theory and experiment for the lowest temporary anion state of $\mathrm{CHCl}_{3}$. The resulting VAE for the ${ }^{2} A_{1}$ anion state of $\mathrm{CCl}_{4}$ is $-1.10 \mathrm{eV}$. Guerra 
TABLE I. Energy-integrated half-peak cross sections and vertical attachment energies.

\begin{tabular}{rccc}
\hline \hline Compound & $\begin{array}{c}\text { Integrated half-peak } \\
\text { cross sections }\left(\mathrm{cm}^{2} \mathrm{eV}\right)\end{array}$ & $\begin{array}{c}\text { VAE } \\
(\mathrm{eV})^{\mathrm{a}}\end{array}$ \\
\hline 1 & Tetrachloromethane & $2.281 \times 10^{-15}$ & -0.34 to $-0.08^{\mathrm{b}}$ \\
2 & Trichloromethane & $4.915 \times 10^{-17}$ & 0.42 \\
3 & $1,1,1$-trichloroethane & $1.236 \times 10^{-16}$ & 0.64 \\
4 & $1,1,2$-trichloroethane & $8.231 \times 10^{-18}$ & 0.8 (est.) \\
5 & 1,1,2-trichloro-2-methylpropane & $2.338 \times 10^{-18}$ & 0.9 (est.) \\
6 & Dichloromethane & $8.020 \times 10^{-20}$ & 1.01 \\
7 & 1,2,3-trichloropropane & $2.653 \times 10^{-18}$ & 1.2 (est.) \\
8 & 1,1-dichloroethane & $6.744 \times 10^{-19}$ & 1.36 \\
9 & 1,1 -dichloropropane & $1.782 \times 10^{-18}$ & 1.39 \\
10 & 1,2-dichloro-2-methylpropane & $3.949 \times 10^{-19}$ & 1.40 \\
11 & 2,2-dichloropropane & $6.138 \times 10^{-19}$ & 1.41 \\
12 & 2,3-dichlorobutane & $2.616 \times 10^{-19}$ & 1.56 \\
13 & 1,2-dichloropropane & $1.907 \times 10^{-19}$ & 1.64 \\
14 & 1,2-dichloroethane & $1.613 \times 10^{-19}$ & 1.70 (est.) \\
15 & 1,3-dichloropropane & $4.298 \times 10^{-20}$ & 1.91 \\
16 & 1,6 -dichlorohexane & $3.086 \times 10^{-20}$ & 2.01 \\
17 & 1,5 -dichloropentane & $7.120 \times 10^{-21}$ & 2.04 \\
18 & 1,4-dichlorobutane & $1.474 \times 10^{-20}$ & 2.07 \\
\hline \hline
\end{tabular}

${ }^{\mathrm{a} R e f e r e n c e} 2$.

${ }^{\mathrm{b}}$ See the text.

et $a .^{20}$ have performed MS-X $\alpha$ calculations, also using stabilization, and predict a VAE of $-0.50 \mathrm{eV}$, with no other adjustments to the experimental results. Both approaches find good agreement between theory and experiment for the temporary anion states of the chloromethanes, and the discrepancy for the stable ${ }^{2} A_{1}$ anion of $\mathrm{CCl}_{4}$ is rather unexpected.

A simpler approach that may be used to estimate VAEs is to compute virtual orbital energies for the LUMOs in a series of related compounds, plot them versus measured VAEs for the accessible temporary anion states, and extrapolate to find the effective VAEs of virtual orbital energies associated with bound anion states. Using a set of such calculations and data for a great number of chloroalkanes, ${ }^{2}$ we previously arrived at a VAE of $-0.34 \mathrm{eV}$ for $\mathrm{CCl}_{4}$. The VAEs used in that study, however, lie relatively high in energy and thus the extrapolation is weighted more heavily by chloroalkanes with relatively few chlorine atoms. Using only the data for $\mathrm{CHCl}_{3}$ and $\mathrm{CH}_{2} \mathrm{Cl}_{2}$, which have relatively low-lying VAEs, the extrapolation yields a VAE of $-0.08 \mathrm{eV}$. We will assume that the VAE of $\mathrm{CCl}_{4}$ is bracketed by these latter two values.

\section{RESULTS}

\section{A. Correlation with VAE}

Our primary objective is to show the connection between the peaks at zero energy and the temporary anion states lying at the VAEs associated with the LUMOs of the chloroalkanes. To do this, we present in Fig. 3 a semilog plot of the integrated half-peak cross sections from our electron beam data as a function of VAE. The filled circles labeled with numbers show the experimental results. The open circles will be discussed later in the paper. The solid line shows the best fit through data points 2-18 excluding compound 6, $\mathrm{CH}_{2} \mathrm{Cl}_{2}$. As mentioned earlier, $\mathrm{CH}_{2} \mathrm{Cl}_{2}$ is the one compound, out of approximately 40 chloroalkanes, that falls well below the best-fit line in our previous study correlating the main DEA cross section peaks with VAE. ${ }^{1}$ The dashed line extends the best-fit solid line down to the range of negative VAEs expected for $\mathrm{CCl}_{4}$, where it intersects at $\mathrm{VAE}=-0.2 \mathrm{eV}$, consistent with the bracketed values. The total variation of the zero peak half-areas is slightly over five orders of magnitude.

$\mathrm{CCl}_{4}$ has properties that differ from those of the remaining chloroalkanes studied here. The leading angular momentum component in an expansion of its $a_{1}$ LUMO wave function in spherical harmonics is $s$ wave; however, the next

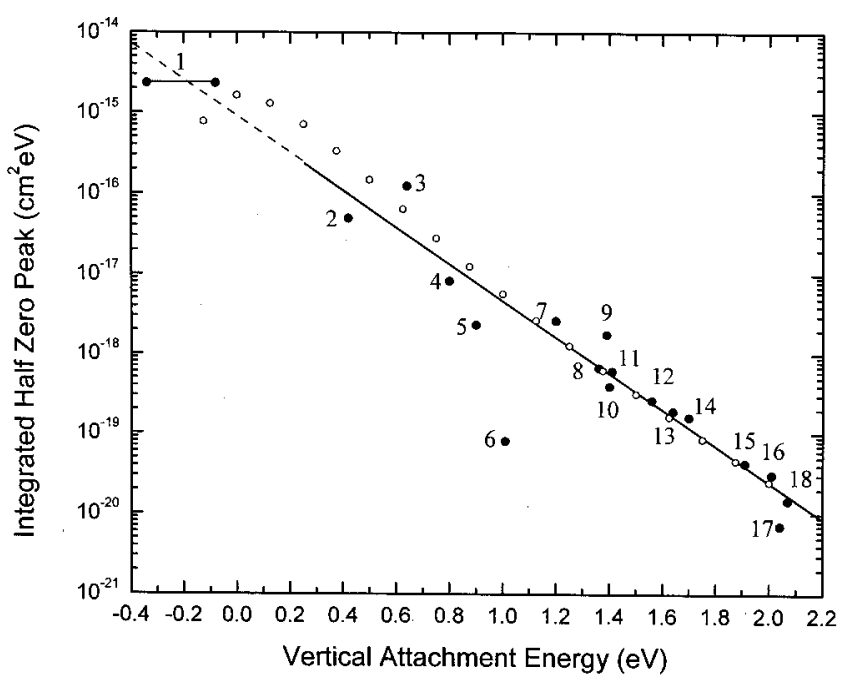

FIG. 3. The $s$ wave cross section peak, integrated from $-0.2 \mathrm{eV}$ to its maximum, as a function of the vertical attachment energy of the compound. The compounds and their reference numbers are listed in Table I. The solid line shows the best-fit line to the data for compounds $2-18$, excluding 6 . The dashed line extends this line to negative vertical attachment energies. Uncertainties in the VAE of compound 1 are indicated by the horizontal line. The open circles show the results of a model computation discussed in Sec. IV C. 


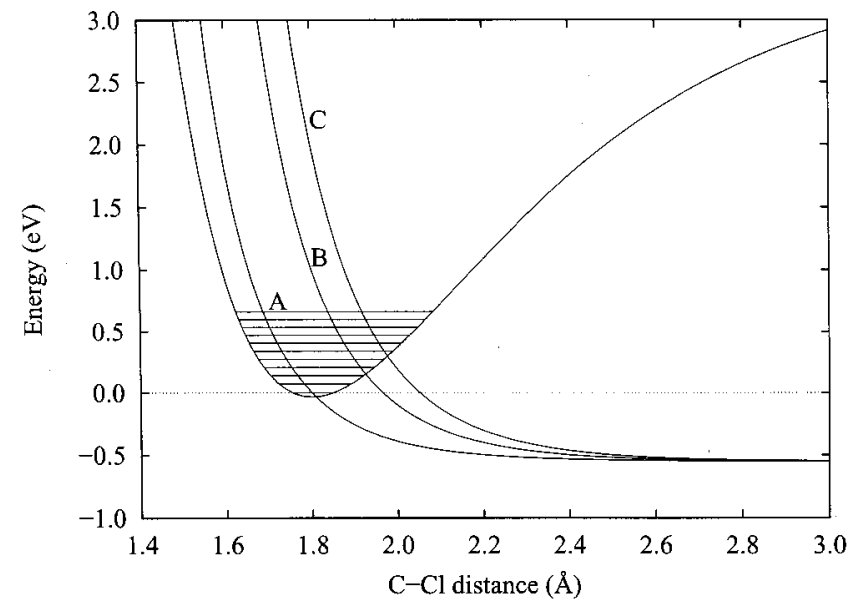

FIG. 4. Representative Morse potential curves for a neutral chloroalkane and anion curves at vertical attachment energies of 0,1 , and $2 \mathrm{eV}$.

higher allowed component is $\ell=3$, rather than the $p$ wave occurring in the other chloroalkanes. Because of the considerable presence of $s$ wave and absence of $p$ and $d$ waves, it could be argued that the ${ }^{2} A_{1}$ state of the $\mathrm{CCl}_{4}$ anion, where it exists in the continuum, does not "qualify" as a resonance because the $s$ wave does not provide a barrier. Indeed, Klar et $a l .{ }^{12}$ have shown that their low energy electron attachment data match predictions of the nonresonant Vogt-Wannier model $^{21}$ within a factor of 2 at sub-meV energies. The monotonic dependence of the integrated half-peak cross sections on VAE shown in Fig. 3, however, suggests that with regard to electron attachment $\mathrm{CCl}_{4}$ differs only in degree from the other chloroalkanes and not in principle, and thus that the mechanism for attachment of near-zero energy electrons is the same in the whole set of compounds. The $a_{1}$ LUMO of $\mathrm{CCl}_{4}$ may be decomposed in spherical harmonics, and we find the following components: $s$ wave (52.6\%), $f$ wave $(26.2 \%), g$ wave $(7.6 \%)$, and the remainder (13.6\%). Because of the substantial admixture of higher partial waves and the angular momentum barriers they reflect, we see no compelling reason to reject a resonance mechanism for $\mathrm{CCl}_{4}$ involving, at least in part, the $a_{1}$ LUMO at low electron energies.

\section{B. Dependence on VAE}

The dependence observed in Fig. 3 implies an exponential decrease in the zero peak magnitude with increasing VAE. For convenience in our discussion, Fig. 4 shows a schematic graph of a Morse potential energy curve for a neutral generic chloroalkane along a $\mathrm{C}-\mathrm{Cl}$ stretch coordinate. A number of the vibrational energies are shown as horizontal lines. We also show examples of three anion potential energy curves with VAEs of 0,1 , and $2 \mathrm{eV}$ to represent different compounds. For simplicity, each of the curves has the same fixed asymptotic energy of $-0.55 \mathrm{eV}$.

An attachment transition to the dissociating anion from the neutral chloroalkane in vibrational level $\nu$ plus a free electron with zero energy is improbable except for levels having energies near that of the crossing of the neutral and anionic potential curves. When the thermal populations of the vibrational levels of the neutral molecule are taken into account, we expect a typical activation process for electron attachment at zero energy. As Fig. 4 indicates, the activation energy will vary with the VAE of the compound.

In the chloroalkanes, the electron affinity of the $\mathrm{Cl}$ atom fixes the difference between the neutral and anion energies at asymptotic $\mathrm{C}-\mathrm{Cl}$ distances. The VAEs are primarily determined, in a rough sense, by three considerations. If we view the anion potential curve as fixed, then variations in the $\mathrm{C}-\mathrm{Cl}$ bond energy among the compounds will alter VAE, with lower bond energies producing lower VAEs. Calculations of bond energies using the concept of isoelectronic isogyric processes ${ }^{22}$ indicate that over the range of compounds from $\mathrm{CH}_{3} \mathrm{Cl}$ to $\mathrm{CHCl}_{3}$, for example, this could account for a decrease in VAE of approximately $0.4 \mathrm{eV}$. On the other hand, the electronic interaction between $\mathrm{C}-\mathrm{Cl} \sigma^{*}$ molecular orbitals as well as inductive effects in the dichloro and trichloro compounds tend to "push" the LUMO to lower energies with increasing chlorination. These effects are much more substantial than the change in bond energies, as illustrated by the VAEs of $\mathrm{CH}_{3} \mathrm{Cl}(3.45 \mathrm{eV})$ and $\mathrm{CHCl}_{3}(0.42$ $\mathrm{eV})$, a change of about $3 \mathrm{eV}$. Finally, the shapes of the anion curves at large separations are also affected by polarization and charge-dipole interactions between $\mathrm{Cl}^{-}$and the remaining radical.

For a given compound with a barrier or activation energy $E_{b}$, the molecular temperature dependence of the zero electron energy attachment process is proportional to $\exp \left[-E_{b} / k T\right]$ from chemical rate theory, ignoring slower varying prefactors. For a fixed gas temperature, as in our experiments, the exponential dependence on VAE observed in Fig. 3 suggests that the $E_{b}$ 's of compounds 2-18 vary linearly with VAE on average. From our data in Fig. 3, $\Delta E_{b} / \triangle V A E \cong 0.135$. Such a simple dependence is rather surprising, considering the number of factors mentioned above that influence the values of VAE.

It is important to note, however, that the scatter from the best-fit line in Fig. 3 is significantly larger than that $(\sim 10 \%)$ in our absolute cross section measurements. ${ }^{1}$ Because of the exponential behavior of the zero peak magnitudes on the precise value of the activation energy, it is likely that the scatter is in fact real and reflects more subtle variations in the potential crossing points that are specific to each of the molecules.

\section{THEORETICAL CONSIDERATIONS}

In this section our goal is to determine the degree to which a simple Morse representation of the potential curves (as shown in Fig. 4) can account for the dependence of the zero peaks on VAE as observed in Fig. 3. To this end, we examine the Franck-Condon factors, thermal populations, and the characteristics of the potential curves.

\section{A. Franck-Condon factors}

The bound Morse curve wave functions used for the Franck-Condon overlaps between the nuclear wave functions of the neutral molecules and the anions formed by electron attachment may all be written exactly and simply in terms of confluent hypergeometric functions. ${ }^{23}$ The con- 


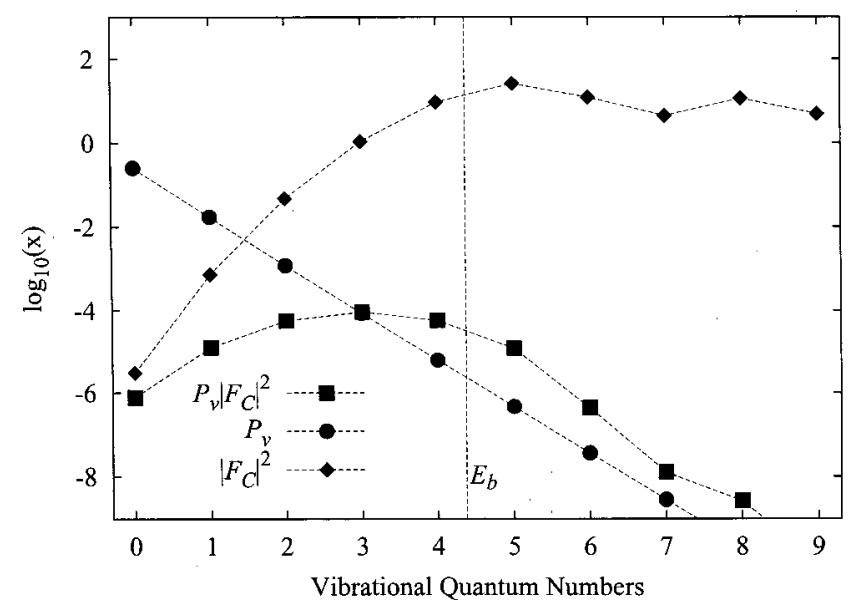

FIG. 5. Computed Franck-Condon factors (filled diamonds), vibrational level populations at $298 \mathrm{~K}$ (filled circles), and their product (filled squares), as a function of vibrational quantum number for an anion potential curve as in curve $\mathrm{C}$ of Fig. 4. The dashed lines serve only to guide the eye. The barrier energy defined by the crossing between the neutral and anion curves lies between $\nu=4$ and 5 and is indicated by the vertical dashed line.

tinuum functions for the overlaps were obtained analytically as phase integral approximations using the Airy function approximation near the classical turning point. ${ }^{24}$ The integrations were carried out numerically rather than employing the commonly used $\delta$ function approximation.

For completeness, we note that our model actually involves an imaginary term in the anion potential to the left of the crossing point with the neutral potential function, reflecting the finite lifetime of the anion against autodetachment of the electron in this region. We have computed FranckCondon overlaps with and without this term, and find that the overall effect is negligible for levels below the crossing energy, and changes those for the first few vibrational levels above the crossing by no more than $10 \%$.

\section{B. Thermal population effects}

The total attachment cross section is the sum of the contributions from the thermal populations of the vibrational levels of the neutral molecule. Therefore

$$
\sigma_{\mathrm{DEA}}=E^{\lambda-1 / 2} C(E) \sum_{v} P_{v}\left|\left\langle\chi_{v}^{b} \mid \chi^{u}\left(E_{\mathrm{ion}}\right)\right\rangle\right|^{2},
$$

where $E$ is the electron energy, $P_{v}$ is the relative population of molecules in the $v$ quantum state, $\chi_{v}^{b}$ is the (bound) neutral molecule wave function, and $\chi^{u}\left(E_{\text {ion }}\right)$ is the (unbound) repulsive-curve ion wave function written as a function of the asymptotic ion energy $E_{\text {ion }}$, which depends upon $v$ and $E$, of course.

Focusing on the repulsive curve labeled " $\mathrm{C}$ " in Fig. 4, for example, we see that it crosses the neutral molecule curve between $v=4$ and 5. From the original classical arguments of Franck, ${ }^{25}$ we would expect molecules in the vibrational levels near $v=5$ to be the most probable for making the transition to the ionic state. The quantum treatment is much less selective, and there is significant probability that several vibrational levels can contribute, either by tunneling or by going "over-the-barrier." Figure 5 shows how these various
TABLE II. Parameters in the Morse potential functions.

\begin{tabular}{llc}
\hline \hline & Bound potential & Unbound potential \\
\hline$D_{e}$ & $3.1 \mathrm{eV}$ & $\ldots$ \\
$\alpha$ & $1.832 \AA^{-1 \mathrm{a}}$ & $6.0 \AA^{-1}$ \\
$r_{e}$ & $1.8 \AA$ & $1.8 \AA$ \\
$\mathrm{V}_{\infty}$ & $3.1 \mathrm{eV}$ & $-0.55 \mathrm{eV}$ \\
\hline \hline
\end{tabular}

${ }^{\text {aAdjusted to give }} \hbar \omega=0.056 \mathrm{eV}$. $(\mu=25 \mathrm{amu})$.

effects interact in a representative case close to the "C" curve in Fig. 4 and at room temperature. The potential curve crossing occurs at a barrier energy of $E_{b}$, lying just above $v=4$ as shown by the vertical dashed line. The 10-base logarithms of $P_{v},\left|\left\langle\chi_{v}^{b} \mid \chi^{u}\left(E_{\text {ion }}\right)\right\rangle\right|^{2}$, and their product are plotted in Fig. 5 versus the quantum number $v$. The lines connecting the points are only to guide the eye. The filled circles giving the Boltzmann population, $P_{v}$, follow a straight line, ignoring vibrational anharmonicity. The Franck-Condon factors, filled diamonds, start small and rise to a peak at the levels near the crossing point. However, the product of the two, filled squares, is maximum at $v=3$ in this example, with the largest contribution arising from tunneling through the narrow top of the barrier. In this specific case the levels below the barrier contribute approximately $70 \%$ of the total. Our model thus indicates that heavy particle tunneling to the anion curve is an important mechanism in the DEA process at thermal electron energies.

Finally, with the results of Fig. 5 in mind, we note that the thermal populations are such that the Franck-Condon factors most affected by the imaginary part of the anionic potential (that is, the levels well above the crossing), are among the least important for determining the DEA cross section. This further justifies our procedure in which we ignore such effects.

\section{VAE dependence of the relative attachment cross sections}

With simple assumptions about the anion potential curves representing the chloroalkanes, we now compute the population-weighted Franck-Condon factors for a series of anion curves with VAEs spanning the range from $2 \mathrm{eV}$ to slightly negative values. The Morse potential function used for the neutral molecule was

$V(r)=D_{e} \exp \left[-\alpha\left(r-r_{e}\right)\right]\left\{\exp \left[-\alpha\left(r-r_{e}\right)\right]-2\right\}+V_{\infty}$.

The expression for the repulsive anion potential took a somewhat different form, as

$$
\begin{aligned}
V(r)= & \frac{E_{a}-V_{\infty}}{3} \exp \left[-\alpha\left(r-r_{e}\right)\right]\left\{\exp \left[-\alpha\left(r-r_{e}\right)\right]+2\right\} \\
& +V_{\infty},
\end{aligned}
$$

where it is assumed that $r_{e}$ will be the same in both expressions. Suitable average values over the various molecules were used to derive the parameters that are listed in Table II. 
TABLE III. Thermal electron attachment rate constants.

\begin{tabular}{rccc}
\hline \hline & & \multicolumn{2}{c}{ Attachment rate constants $\left(\mathrm{cm}^{3} / \mathrm{s}\right)$} \\
\cline { 3 - 4 } & Compound & Electron beam & Swarm studies \\
\hline 1 & Tetrachloromethane & $2.80 \times 10^{-7}$ & $3.6-3.9 \times 10^{-7} \mathrm{a}$ \\
2 & Trichloromethane & $5.41 \times 10^{-9}$ & $3.6-4.7 \times 10^{-9} \mathrm{~b}$ \\
3 & $1,1,1$-trichloroethane & $2.2 \times 10^{-9}$ & $1.1-1.6 \times 10^{-8} \mathrm{c}$ \\
4 & $1,1,2$-trichloroethane & $2.90 \times 10^{-10}$ & $1.5-3.1 \times 10^{-10 \mathrm{~d}}$ \\
5 & 1,1,2-trichloro-2-methylpropane & $\approx 1.6 \times 10^{-11}$ & $4.6-4.8 \times 10^{-12 \mathrm{e}}$ \\
6 & Dichloromethane & $6.31 \times 10^{-10}$ & \\
7 & 1,2,3-trichloropropane & $6.87 \times 10^{-11}$ & $2.1 \times 10^{-11 \mathrm{f}}$ \\
8 & 1,1-dichloroethane & $3.15 \times 10^{-10}$ & \\
9 & 1,1-dichloropropane & $4.80 \times 10^{-11}$ & \\
10 & 1,2-dichloro-2-methylpropane & $5.73 \times 10^{-11}$ & \\
11 & 2,2-dichloropropane & $3.11 \times 10^{-11}$ & \\
12 & 2,3-dichlorobutane & $2.70 \times 10^{-11}$ & \\
13 & 1,2-dichloropropane & $4.72 \times 10^{-11}$ & $3.2 \times 10^{-11 \mathrm{f}}$ \\
14 & 1,2-dichloroethane & $4.94 \times 10^{-12}$ & \\
15 & 1,3-dichloropropane & $4.00 \times 10^{-12}$ & \\
16 & 1,6-dichlorohexane & $9.00 \times 10^{-13}$ & \\
17 & 1,5-dichloropentane & $2.80 \times 10^{-12}$ & \\
18 & 1,4-dichlorobutane & & \\
\hline
\end{tabular}

${ }^{a}$ References 30-32.

${ }^{\mathrm{b}}$ References 30, 33.

${ }^{c}$ References 34, 35.

${ }^{\mathrm{d}}$ References 35-37.

${ }^{\mathrm{e}}$ References 38, 39.

${ }^{\mathrm{f}}$ Reference 35 .

For simplicity, the anion curves, three of which are shown in Fig. 4, have a fixed asymptotic energy and are purely repulsive at all distances.

The population weighted Franck-Condon factors are shown as open circles in Fig. 3. These relative values are normalized to the earlier best-fit line at $\mathrm{VAE}>1.5 \mathrm{eV}$. Considering the simplicity of the approximations to the anion curves, the results are quite satisfactory, yielding essentially all of the change in magnitude of the cross section as a function of VAE. For $\mathrm{VAE}<1 \mathrm{eV}$, the open circles lie above the earlier best-fit straight line. This is traceable to the barrier potential declining somewhat faster than linearly with VAE in this regime with our choice of potential curves. Unfortunately, the experimental measurements cannot help to resolve this issue because of the absence of compounds having VAEs between 0 and $0.4 \mathrm{eV}$. Introduction of additional parameters and further empirical adjustment of the potential curves would undoubtedly improve the agreement, but our simple approximations capture the main "physics" of the zero peak dependence on VAE.

A more rigorous theoretical treatment of DEA and the role of the crossing point and vibrational populations in $\mathrm{CH}_{3} \mathrm{Cl}, \mathrm{CH}_{3} \mathrm{Br}$ and $\mathrm{CH}_{3} \mathrm{I}$ can be found in work by Wilde et al. ${ }^{26}$

\section{ATTACHMENT RATE CONSTANTS FROM ELECTRON BEAM DATA}

\section{A. Determination of attachment rate constants}

The thermal electron attachment rate constant as a function of electron energy, $E$, is given by ${ }^{27}$

$$
k=\frac{\sqrt{8}}{\sqrt{m_{e} \pi}\left(k_{B} T\right)^{3 / 2}} \int_{0}^{\infty} \sigma(E) E \exp \left(\frac{-E}{k_{B} T}\right) d E .
$$

The model function we use for the low energy cross section is given by Eq. (2), but this can be valid only for energies between 0 and $\approx 0.15 \mathrm{eV}$. To take the higher energy parts of the cross section into account, we have calculated the rate constants using the analytic expression for low energies and the measured cross section above this range. This procedure assumes that the part of the cross section where the $p$ wave is dominant is sufficiently slowly varying so that the instrumental width function is unimportant. Thus, our expression for the rate constant is

$$
\begin{aligned}
k= & \left(\sqrt{\frac{8}{m_{e} \pi}}\right)\left[A_{1} \gamma\left(a+2, e_{c}\right)\left(k_{B} T\right)^{a+1 / 2}+A_{2} \gamma\left(a+3, e_{c}\right)\right. \\
& \left.\times\left(k_{B} T\right)^{a+3 / 2}+B_{1} \gamma\left(b+2, e_{c}\right)\left(k_{B} T\right)^{b+1 / 2}\right]+Q_{\text {num }},
\end{aligned}
$$

where $\gamma(a, x)$ is the conventional partial $\Gamma$-function, ${ }^{28} Q_{\text {num }}$ is the numerically integrated region directly using the experimental data, and $e_{c}$ is a point near the lower end of the $p$ wave contribution where the analytic fit and the experimental data are equal. In all cases except $\mathrm{CH}_{2} \mathrm{Cl}_{2}$ and 1,1,2trichloroethane such a point exists, and in the case of the latter the two functions are within experimental error of one another. The rate constant for $\mathrm{CH}_{2} \mathrm{Cl}_{2}$ is only approximate.

The electron energy distribution we employ is characterized by a temperature of $298 \mathrm{~K}$. Because the low-energy electron scattering is largely dominated by the zero energy attachment peak in the chloroalkanes, we expect that the thermal attachment rate constant may also reflect a monotonic dependence on the VAEs of the molecules. Table III 


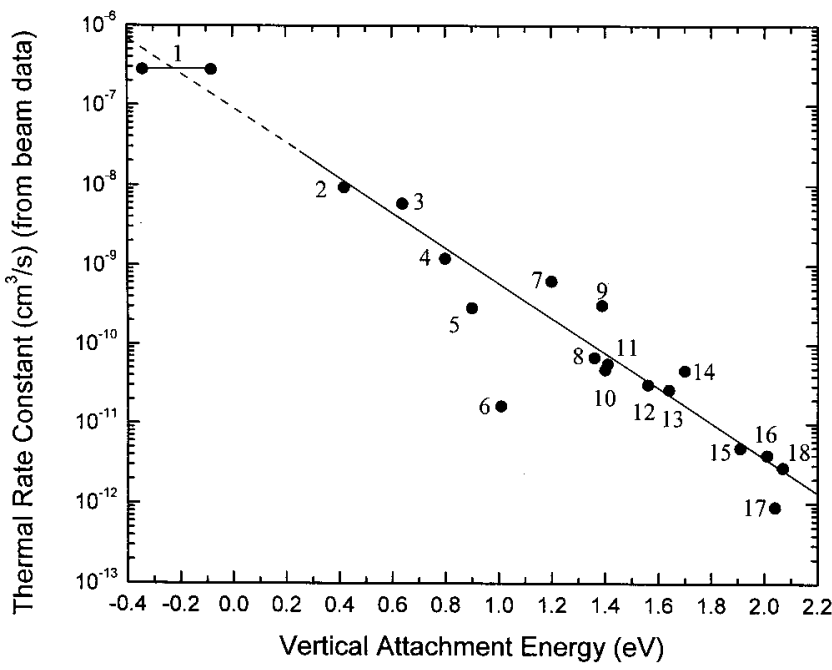

FIG. 6. Thermal attachment rate constants (298 K) computed from electron beam cross section measurements as a function of vertical attachment energy. The solid line is the best-fit line through the data for compounds $2-18$, excluding 6 . The dashed line extends the solid line to negative values of VAE.

displays the thermal rate constants derived from the present electron beam data along with values determined by swarm and other techniques. The computed attachment rate constants are plotted on a semilog scale as a function of VAE in Fig. 6. The solid line is a best fit to compounds $2-18$, omitting 6 as before. The dashed line shows the extension to negative VAEs. Again, the result for $\mathrm{CCl}_{4}$ is consistent with the best-fit line determined from 2-18. As in Fig. 3, a reasonable correlation is observed over more than 5 orders of magnitude in the rate constant. Christophorou ${ }^{29}$ has previously noted a precipitous decline in rate constant with increasing energy of the attaching state in halocarbons. The existence of a consistent set of VAEs has enabled us to refine this behavior in the present work. We note that the differences in the relative positions of several of the compounds from those shown in Fig. 3 can be traced to the relative sizes of the $p$ wave portions of the cross sections. In the highenergy wings of the electron energy distribution, these contributions play a greater role.

\section{B. Comparisons of rate constants}

In high resolution studies of electron attachment such as that of Klar et al. ${ }^{12}$ and by others, thermal attachment rate constants have been employed to put relative DEA cross sections on an absolute scale. In the previous section we did the reverse and computed the rate constants from our absolute beam measurements. We caution the reader that this process is intrinsically less reliable because of the uncertainties of the zero-peak fitting procedure and the sensitivity of the rate constant to the energy exponent in Eq (2). Nevertheless, we compare them here against rates determined by swarm and other techniques.

Unfortunately, thermal attachment rate constant data exist for only seven of the chloroalkanes in our study, to our knowledge. A recent compilation of rates is given by Christophorou. ${ }^{29}$ A variety of techniques has been employed

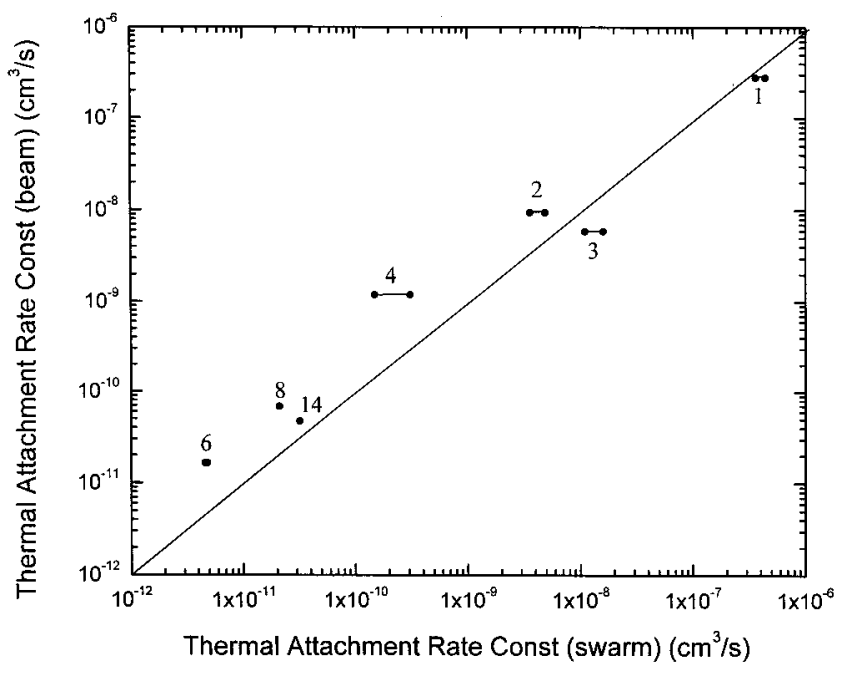

FIG. 7. Thermal attachment rate constants (298 K) computed from electron beam cross section measurements plotted against values derived directly from swarm and other techniques. The diagonal line shows where data with perfect agreement would lie. As described in the text, an estimated range of "swarm" values is shown by the bracketed values.

in such studies and it is beyond our purview to select from these the most reliable values. Rather, we have chosen a representative range of values for those compounds that have been extensively studied, tending to favor more recent work, and entered these in Table III and plotted them in Fig. 7. For example, for compound $1, \mathrm{CCl}_{4}$, which has been studied most extensively, Christophorou ${ }^{29}$ lists values ranging from $1.3-4.4 \times 10^{-7} \mathrm{~cm}^{3} \mathrm{~s}^{-1}$ with an average over all of 3.4 $\times 10^{-7} \mathrm{~cm}^{3} \mathrm{~s}^{-1}$. We choose a reduced range $\mathrm{s}^{30-32}$ from $3.6-3.9 \times 10^{-7} \mathrm{~cm}^{3} \mathrm{~s}^{-1}$. In similar fashion for $\mathrm{CHCl}_{3}$, compound 2, we select $30,33 \quad 3.6-4.7 \times 10^{-9} \mathrm{~cm}^{3} \mathrm{~s}^{-1}$. Fewer measurements ${ }^{34,35}$ have been carried out in compound 3 , $1,1,1-\mathrm{C}_{2} \mathrm{H}_{3} \mathrm{Cl}_{3}, 1.1-1.6 \times 10^{-8} \mathrm{~cm}^{3} \mathrm{~s}^{-1}$, compound $4,{ }^{35-37}$ $1,1,2-\mathrm{C}_{2} \mathrm{H}_{3} \mathrm{Cl}_{3}, \quad 1.5-3.1 \times 10^{-10} \mathrm{~cm}^{3} \mathrm{~s}^{-1}$ and compound $6,{ }^{38,39} \mathrm{CH}_{2} \mathrm{Cl}_{2}, 4.6-4.8 \times 10^{-12} \mathrm{~cm}^{3} \mathrm{~s}^{-1}$. Only a single study $^{35}$ has been published in compounds $8,1,1-\mathrm{C}_{2} \mathrm{H}_{4} \mathrm{Cl}_{2}$ and $14,1,2-\mathrm{C}_{2} \mathrm{H}_{4} \mathrm{Cl}_{2}$.

Figure 7 shows the rate constants derived from our beam measurements versus those determined by other techniques, labeled generically as "swarm," on the $x$ axis. The diagonal solid line shows where data indicating perfect agreement would lie. Overall the agreement is rather disappointing, but there are a number of experimental caveats of which to be aware. Each of the convoluted zero peaks is put on an absolute scale by reference to the cross section for the $p$ wave DEA feature lying near the VAE. The latter cross sections are determined $^{1}$ in a static cell maintained near $65^{\circ} \mathrm{C}$ and have errors of approximately $\pm 10 \%$. The magnitude of the zero peak relative to that of the $p$ wave peak, however, is determined in a crossed electron/molecular beam experiment in which the molecular temperature is close to $298 \mathrm{~K}$, and the anion fragments are transported to a multichannel plate and counted. The difference in temperature will likely cause the zero peaks to be slightly overestimated. A more significant error could arise from kinetic discrimination against the more energetic ions produced via the $p$ wave peak relative to 
those of the zero peak. This too will cause the zero peak cross section to appear larger than it should, and one would expect this to be more pronounced in compounds with higher VAEs. In the context of this discussion, we note that there was no evidence that the target gas in the background contained a hotter component owing to heating by the filament. This was tested by diverting the gas from the molecular beam and injecting it directly into the vacuum chamber. We also mention that the size of the zero peak relative to the $p$ wave peak and the zero peak shape are, of course, sensitive to the electron beam distribution and vary with the tuning of the apparatus. The primary results of the work as seen in Figs. 3, 6, and 7, however, are based on fits to the cross section convoluted with the electron distribution, and the areas under the zero peaks are relatively insensitive to tuning.

Errors associated with the fits to the convoluted zero peaks and the calculations of the rate constants are difficult to estimate. Existing data in $\mathrm{CCl}_{4}$, however, allow us to explore our fitting procedure in somewhat more detail. The rate constant for $\mathrm{CCl}_{4}\left(3.79 \times 10^{-7} \mathrm{~cm}^{3} / \mathrm{s}\right)$ determined by Orient et al. ${ }^{31}$ is cited with an error of $\pm 8 \%$, believed to be "conservative" by its authors, and is arguably the most accurately determined rate constant of the compounds studied here. The rate constant determined from our electron beam measurement is $2.80 \times 10^{-7} \mathrm{~cm}^{3} / \mathrm{s}$, lower by $26 \%$. The parametrization of Eq. (2) uses only a single energy exponent, $a$, for the diverging portion of the cross section as electron energy goes to zero. In our $\mathrm{CCl}_{4}$ fit $a=-0.657$. The high resolution laser photoattachment data of Klar et al., ${ }^{12}$ on the other hand, show that this exponent decreases smoothly from $\approx-1$ at $50 \mathrm{meV}$ to $\approx-0.5$ at $1 \mathrm{meV}$. Our procedure thus averages over this changing exponent, a potential source of error. To determine if the error is substantial, we used the empirical expression given by Klar et al. ${ }^{12}$ to fit their data [Eq. (7) and Table I of Ref. 12] and convoluted it with the electron distribution of our apparatus. To reach agreement with our measured zero energy peak, it was necessary to scale our data up by a factor of 1.3. Our convoluted cross section is thus $23 \%$ below that of Klar et al., consistent with the difference in the attachment rates.

Unfortunately, our DEA cross section for $\mathrm{CCl}_{4}$ was not measured in the total ion collection apparatus used for the remaining compounds. Rather, it was done indirectly in the crossed-beam apparatus by reference to the cross sections for $\mathrm{CH}_{2} \mathrm{Cl}_{2}$ and $\mathrm{CHCl}_{3}$. In the first case, a cross section of $4.09 \times 10^{-16} \mathrm{~cm}^{2}$ at the $0.8 \mathrm{eV}$ maximum was found. In the second, a value of $4.95 \times 10^{-16} \mathrm{~cm}^{2}$ was determined. These have been averaged to give $4.5 \times 10^{-16} \mathrm{~cm}^{2} \pm 15 \%$. Taking the rate constant of Orient et al. ${ }^{31}$ at face value would require increasing our cross section by about $8 \%$ to cause the relative error bars to begin to overlap. In view of the nonstandard calibration we employed, this is not unreasonable. The rate constants for the remaining compounds $2-4,6,8$, and 14 , however, lie well outside our anticipated errors.

We conclude this section with a few comments regarding activation energies and the electron attachment process. As shown earlier, the slope of the best-fit line in Fig. 3, as well as that in Fig. 6, indicates that the difference in effective crossing-point energies of the neutral and anion potential curves for two chloroalkanes can be related to the difference in their VAEs, that is, $\Delta E_{b}=0.135 \cdot \Delta V A E$, on average. Our model potential curves, such as those shown in Fig. 4, indicate that the crossing energy reaches that of the ground vibrational level at $\mathrm{VAE} \approx 0.25 \mathrm{eV}$, that is, $E_{b}=0$ for $0<\mathrm{VAE}$ $<0.25 \mathrm{eV}$. The activation energy for compounds with VAE $>0.25 \mathrm{eV}$ is thus given approximately by

$$
E_{b} \cong 0.135(V A E-0.25) \text {. }
$$

This expression will be more accurate for large VAE than for small values. We should also comment that $E_{b}$ here is an effective barrier energy. Because of the tunneling that takes place from vibrational levels below the barrier, the actual crossing lies approximately $50 \mathrm{meV}$ higher, according to our model calculations, for $\mathrm{VAE} \geqslant 1.0 \mathrm{eV}$.

The predicted activation energies from Eq. (8) cannot be directly compared to those determined from Arrhenius plots by the usual means. The latter are derived from attachment experiments in which molecular and electron temperatures are in equilibrium at each temperature. Although it is sometimes assumed that the slopes determined from these plots yield the crossing energy of the potential curves, it is clear from the strong energy dependence of the DEA cross sections at low energies that changes in the electron temperature will also vary the attachment rates.

To illustrate the difference between these quantities, the VAE of $\mathrm{CHCl}_{3}(0.42 \mathrm{eV})$ implies a molecular activation barrier of $0.023 \mathrm{eV}$ from Eq. (8). Although this value is only approximate because of the low VAE, it is clearly much less than the activation energy $(0.13 \pm 0.01 \mathrm{eV})$ determined by Sunagawa and Shimamori ${ }^{40}$ from an Arrhenius plot of the attachment rate constants. As these authors and others have shown, the rate constant as a function of the electron temperature increases dramatically for these molecules held at $300 \mathrm{~K}$. This effect thus appears to dominate the slope of the Arrhenius plot. From our electron scattering measurements, it is clear that this behavior is a consequence of the low energy of the $p$ wave peak in the DEA cross section.

\section{SUMMARY}

In this work we have shown that the zero energy peaks appearing in the DEA cross sections of the chloroalkanes display a systematic dependence on the VAE associated with electron attachment into the LUMO. At the heart of this behavior is the essentially linear relationship between VAE and the energy of the crossing point between the neutral and anion potential curves. Taken together with our earlier work ${ }^{1}$ showing the correlation between the peak $p$ wave DEA cross sections and VAE, this is a second illustration of the strong "family resemblance" of the DEA process in the chloroalkanes. We note that $\mathrm{CH}_{2} \mathrm{Cl}_{2}$ is anomalous in both correlations.

The relative zero peak behavior was justified within a local-potential resonance treatment of DEA using simple Morse potentials. Franck-Condon factors for the attachment process indicated that a substantial fraction of the total attachment arises from heavy particle tunneling through the narrow peak of the barrier. 
TABLE IV. Parameters for model expression to represent cross sections.

\begin{tabular}{lccccc}
\hline \hline \multicolumn{1}{c}{ Compound } & $A_{1}$ & $a$ & $B_{1}$ & $b$ & $A_{2}$ \\
\hline Tetrachloromethane & $1.04 \times 10^{-1}$ & -0.657 & $-2.29 \times 10^{-1}$ & 1.34 & $6.83 \times 10^{-1}$ \\
Trichloromethane & $4.24 \times 10^{-2}$ & -0.709 & $8.73 \times 10^{-1}$ & 0.436 & 0.000 \\
1,1,1-trichloroethane & $5.53 \times 10^{-3}$ & -0.945 & $1.57 \times 10^{-1}$ & 0.495 & $1.54 \times 10^{-2}$ \\
1,1,2-trichloroethane & $1.06 \times 10^{-2}$ & -0.886 & 2.257 & 0.665 & $2.44 \times 10^{-1}$ \\
1,1,2-trichloro-2-methylpropane & $7.11 \times 10^{-4}$ & -0.989 & 2.058 & 0.579 & $2.01 \times 10^{-1}$ \\
Dichloromethane & $5.37 \times 10^{-5}$ & -0.998 & 1.666 & 0.402 & $6.12 \times 10^{-2}$ \\
1,2,3-trichloropropane & $2.77 \times 10^{-2}$ & -0.755 & 2.142 & 0.530 & $1.46 \times 10^{-2}$ \\
1,1-dichloroethane & $1.76 \times 10^{-2}$ & -0.849 & $3.01 \times 10^{-1}$ & 0.585 & 0.000 \\
1,1-dichloropropane & $7.25 \times 10^{-2}$ & -0.689 & $5.10 \times 10^{-7}$ & 0.429 & $3.91 \times 10^{-1}$ \\
1,2-dichloro-2-methylpropane & $4.01 \times 10^{-3}$ & -0.951 & 2.842 & 0.760 & $1.41 \times 10^{-1}$ \\
2,2-dichloropropane & $1.58 \times 10^{-2}$ & -0.871 & $2.49 \times 10^{-1}$ & 0.348 & $1.48 \times 10^{-3}$ \\
2,3-dichlorobutane & $8.15 \times 10^{-5}$ & -0.999 & 2.856 & 0.674 & $1.31 \times 10^{-1}$ \\
1,2-dichloropropane & $3.14 \times 10^{-6}$ & -1.000 & 1.863 & 0.512 & $1.50 \times 10^{-1}$ \\
1,2-dichloroethane & $9.87 \times 10^{-2}$ & -0.517 & 2.314 & 0.585 & 0.000 \\
1,3-dichloropropane & $1.03 \times 10^{-2}$ & -0.910 & $6.69 \times 10^{-1}$ & 0.263 & $1.14 \times 10^{-1}$ \\
1,6-dichlorohexane & $4.42 \times 10^{-2}$ & -0.773 & $8.43 \times 10^{-1}$ & 0.794 & $6.34 \times 10^{-4}$ \\
1,5-dichloropentane & $2.06 \times 10^{-2}$ & -0.852 & $6.43 \times 10^{-1}$ & 0.446 & $4.50 \times 10^{-2}$ \\
1,4-dichlorobutane & $7.06 \times 10^{-2}$ & -0.648 & 1.143 & 0.521 & $3.53 \times 10^{-6}$ \\
\hline \hline
\end{tabular}

Attachment rate constants computed from our electron beam studies also display an exponential decrease with increasing VAE. Although they generally track rate constants measured more directly by swarm and other techniques over five orders of magnitude, they disagree individually by amounts that appear to be outside the error limits, except for $\mathrm{CCl}_{4}$. Internally consistent sets of rate constants for more of the chloroalkanes would be valuable in tracking the source of the disagreement, as would careful measurements of the zero peaks with higher energy resolution electron beams. The absolute cross sections determined previously ${ }^{1}$ for the $p$ wave peaks in these compounds should be useful for putting the zero peaks on an absolute scale, as employed here.

\section{ACKNOWLEDGMENTS}

This work was supported by NSF and USDA/CSREES. The authors are grateful to Ilya Fabrikant for many useful conversations on DEA and threshold laws.

\section{APPENDIX A: THRESHOLD PROPERTIES OF THE DEA CROSS SECTION}

The basic form of the cross section for DEA (when exothermic) follows from the definition ${ }^{41}$ of the cross section for inelastic processes

$$
\sigma_{\mathrm{DEA}}=\frac{k_{I}}{k_{e}}\left\langle\left|f\left(\hat{k}_{e}, \Omega\right)\right|^{2}\right\rangle_{\Omega},
$$

where $k_{I}$ and $k_{e}$ are, respectively, the asymptotic ion and electron momenta, $f$ is the inelastic scattering amplitude, $\Omega$ symbolizes coordinates establishing the orientation of the molecule, and \langle\rangle$_{\Omega}$ indicates an average over all molecule orientations. Restricting the argument to exothermic cases makes $k_{I}$ essentially constant as $E$ and $k_{e}$ go to zero, and if $f$ is also well behaved (remains finite) as $E$ goes to zero, we obtain the classic Bethe 1/v law for exothermic reactions.

Although we would not expect quantitative results, we may obtain useful information concerning $f$ from the

TABLE V. Instrument parameters and fitting criteria.

\begin{tabular}{|c|c|c|c|c|c|}
\hline Compounds & $\Delta E_{0}, \mathrm{eV}$ & $\Gamma_{i}, \mathrm{eV}$ & $A_{i}$ & rms Dev. & Num pts \\
\hline Tetrachloromethane & 0.009 & 0.095 & 0.294 & 0.004 & 25 \\
\hline Trichloromethane & 0.000 & 0.067 & 0.262 & 0.006 & 56 \\
\hline 1,1,1-trichloroethane & 0.005 & 0.084 & 0.414 & 0.003 & 64 \\
\hline 1,1,2-trichloroethane & 0.018 & 0.102 & 0.328 & 0.003 & 63 \\
\hline 1,1,2-trichloro-2-methylpropane & 0.001 & 0.090 & 0.332 & 0.003 & 63 \\
\hline Dichloromethane & 0.014 & 0.114 & 0.220 & 0.003 & 63 \\
\hline 1,2,3-trichloropropane & 0.010 & 0.093 & 0.311 & 0.002 & 63 \\
\hline 1,1-dichloroethane & 0.007 & 0.070 & 0.358 & 0.002 & 103 \\
\hline 1,1-dichloropropane & 0.013 & 0.108 & 0.362 & 0.001 & 83 \\
\hline 1,2-dichloro-2-methylpropane & 0.007 & 0.088 & 0.370 & 0.003 & 63 \\
\hline 2,2-dichloropropane & -0.001 & 0.084 & 0.379 & 0.002 & 82 \\
\hline 2,3-dichlorobutane & 0.003 & 0.093 & 0.381 & 0.003 & 63 \\
\hline 1,2-dichloropropane & -0.001 & 0.086 & 0.229 & 0.004 & 63 \\
\hline 1,2-dichloroethane & 0.032 & 0.094 & 0.252 & 0.002 & 46 \\
\hline 1,3-dichloropropane & -0.001 & 0.116 & 0.354 & 0.003 & 84 \\
\hline 1,6-dichlorohexane & 0.012 & 0.105 & 0.413 & 0.003 & 63 \\
\hline 1,5-dichloropentane & 0.020 & 0.101 & 0.464 & 0.004 & 62 \\
\hline 1,4-dichlorobutane & 0.006 & 0.091 & 0.257 & 0.003 & 62 \\
\hline
\end{tabular}


TABLE VI. Scale factors.

\begin{tabular}{lc}
\hline \multicolumn{1}{c}{ Compound } & Scale, $\mathrm{cm}^{2}$ \\
\hline Tetrachloromethane & $3.20 \times 10^{-14}$ \\
Trichloromethane & $1.18 \times 10^{-15}$ \\
1,1,1-trichloroethane & $2.53 \times 10^{-15}$ \\
1,1,2-trichloroethane & $1.56 \times 10^{-16}$ \\
1,1,2-trichloro-2-methylpropane & $4.73 \times 10^{-17}$ \\
Dichloromethane & $2.86 \times 10^{-18}$ \\
1,2,3-trichloropropane & $7.14 \times 10^{-17}$ \\
1,1-dichloroethane & $1.53 \times 10^{-17}$ \\
1,1-dichloropropane & $3.03 \times 10^{-17}$ \\
1,2-dichloro-2-methylpropane & $8.29 \times 10^{-18}$ \\
2,2-dichloropropane & $1.20 \times 10^{-17}$ \\
2,3-dichlorobutane & $5.73 \times 10^{-18}$ \\
1,2-dichloropropane & $4.62 \times 10^{-18}$ \\
1,2-dichloroethane & $4.14 \times 10^{-18}$ \\
1,3-dichloropropane & $7.06 \times 10^{-19}$ \\
1,6-dichlorohexane & $4.91 \times 10^{-19}$ \\
1,5-dichloropentane & $1.34 \times 10^{-19}$ \\
1,4-dichlorobutane & $2.88 \times 10^{-19}$ \\
\hline \hline
\end{tabular}

distorted-wave Born approximation. Following $\mathrm{Wu}$ and Ohmura, ${ }^{41}$ we have for the scattering amplitude in the $v$ th state

$$
f_{v}=-(4 \pi)^{-1}\left\langle\psi_{I}^{(-)}|U| \psi_{\nu}^{(+)}\right\rangle
$$
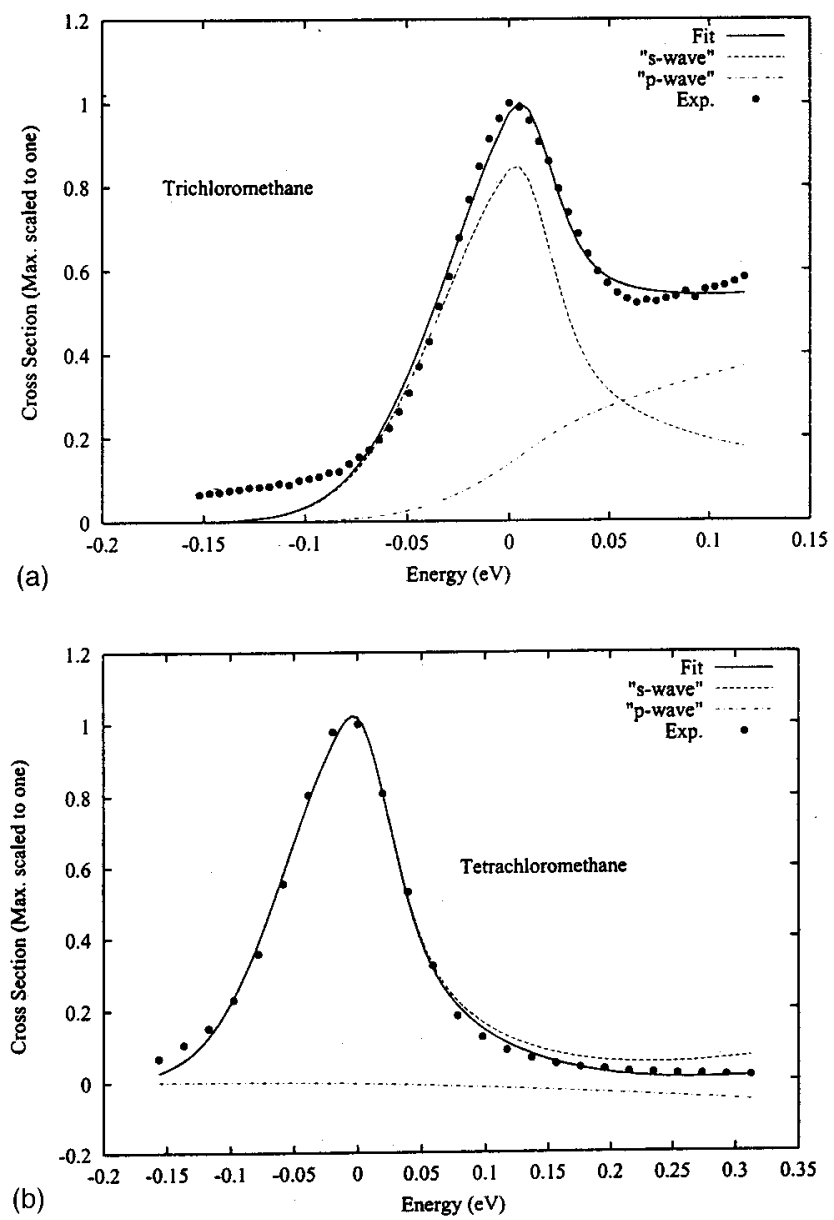

FIG. 8. As in Fig. 2 for trichloromethane and tetrachloromethane.
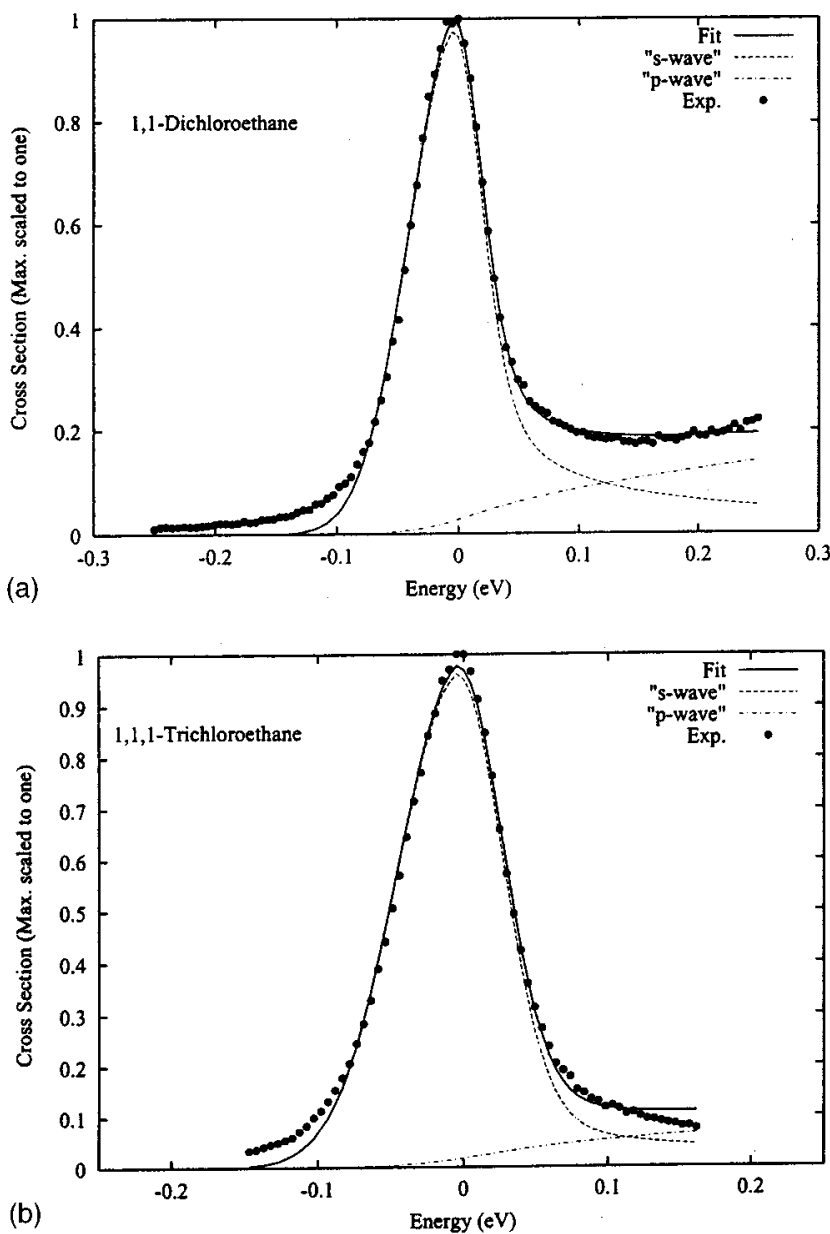

FIG. 9. As in Fig. 2 for 1,1-dichloroethane and 1,1,1-trichloroethane.

where $\psi_{\nu}^{(+)}$is the wave function for the neutral molecule in the $\nu$ vibrational state, and the ion function must have incoming wave boundary conditions. Using the BornOppenheimer approximation in the usual way, assuming that nuclear coordinate dependent electronic integrals can be removed from integrals over nuclear coordinates, we obtain the expression we used in Sec. II A

$$
\sigma_{\mathrm{DEA}}=E^{\lambda-1 / 2} C(E) \sum_{v} P_{v}\left|\left\langle\chi_{v}^{b} \mid \chi^{u}\left(E_{\mathrm{ion}}\right)\right\rangle\right|^{2} .
$$

In this expression we have absorbed all numerical constants into the quantity $C$, replaced $k_{e}$ by $E^{1 / 2}$ to which it is proportional, and written the threshold behavior of $|f|^{2}$ as $E^{\lambda} C(E)$, where $C$ is finite at $E=0$.

The exact behavior we obtain for our various molecules depends upon the threshold properties of the quantity $C(E)$. A number of cases are possible:

(a) $C$ might accidently be zero at $E=0$. This is unlikely.

(b) $\lambda=0$ yields the classic case of the Bethe $1 / v$ law $^{10}$ for inelastic reactions. With our cross sections, this is expected for molecules with no permanent electric dipole moment. We point out that several of the larger molecules in this study have multiple conformers, including the possibility that some are with and some are without electric moments. 


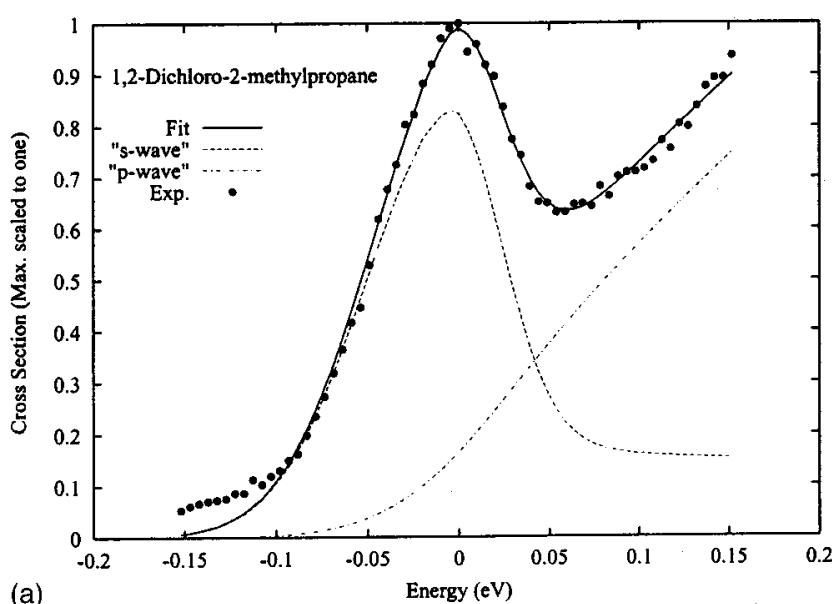

(a)

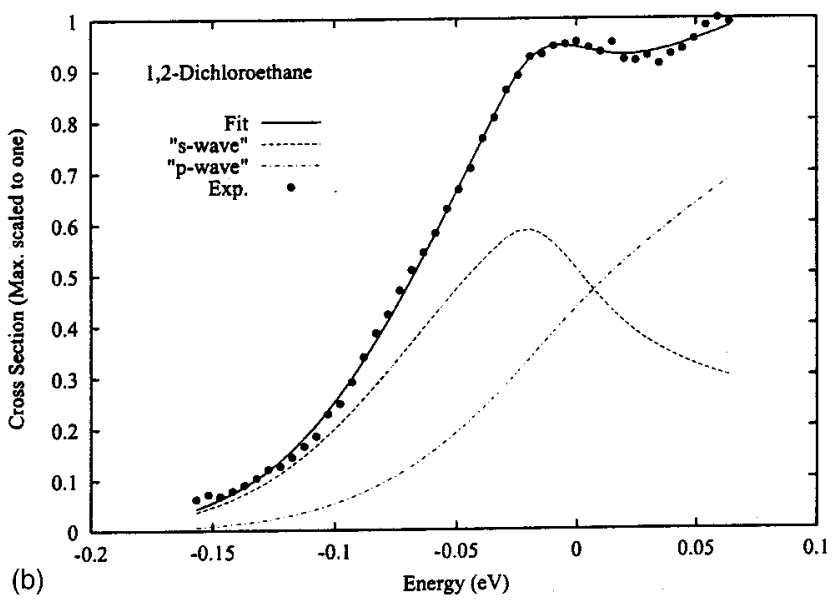

FIG. 10. As in Fig. 2 for 1,2-dichloroethane and 1,2-dichloro-2methylpropane.

(c) $-1 / 2<\lambda<0$ occurs with molecules or conformers that have subcritical $(<1.62 \mathrm{D})$ electric moments. This, however, pertains only if the electron velocity is fast compared to the rotation of the molecular states populated. Thus, at very low energies the situation reverts to the Bethe law.

(d) $\lambda=-1 / 2$ and $C$ oscillating occurs with supercritical electric moments. There is again reversion to the Bethe law at the lowest of electron energies.

Some modification of these results is expected if quadrupole moments and molecular polarizability are considered, but we ignore these complications. We do note, however, that for very slow electrons, the rotation of polar molecules merely becomes part of the polarization interaction between the electron and the molecule.

\section{APPENDIX B: FITTING PARAMETERS}

Table IV summarizes the parameters used for fitting our model expression to the measured zero energy peaks near threshold. Table $\mathrm{V}$ lists the parameters characterizing the electron beam energy distribution and fitting criteria. Table VI lists the scale factors required to put the unity-normalized zero peaks on an absolute cross section scale. Figures 8-11
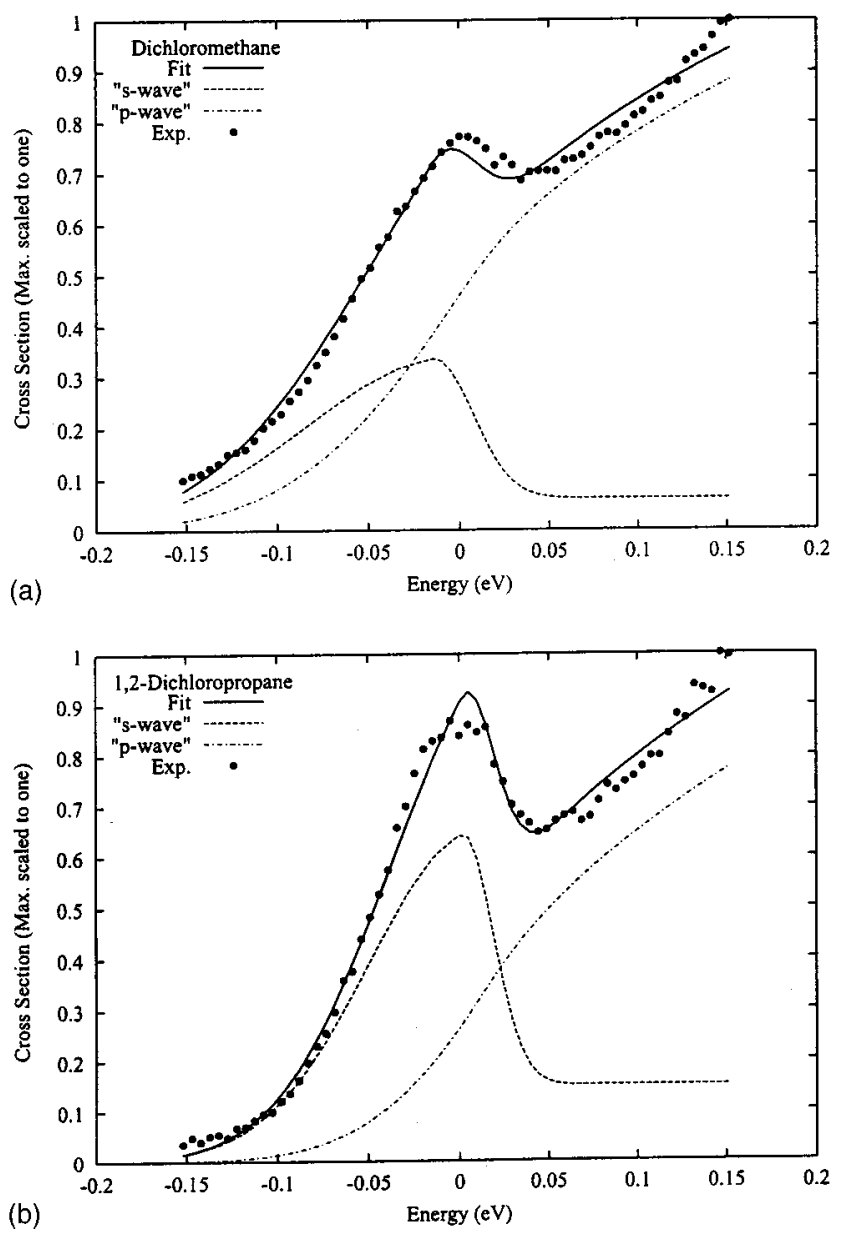

FIG. 11. As in Fig. 2 for dichloromethane and 1,2-dichloropropane, illustrating the poorest fits obtained.

illustrate the fitting in a sample of the remaining chloroalkanes. Figure 8 shows the results in trichloromethane and tetrachloromethane. The data for the latter compound were measured considerably earlier ${ }^{17}$ and on a coarser energy scale. Spline interpolations were used to improve the quality of the fit. The negative sign for parameter $B_{1}$ in Table IV is not physically meaningful.

Figure 9 illustrates two compounds in which the attachment is dominated by the zero energy peak. Figure 10 displays results in two compounds in which the rapidly rising $p$-wave contribution plays a more significant role. Finally, Fig. 11 shows the results for the two compounds with the poorest quality fits, $\mathrm{CH}_{2} \mathrm{Cl}_{2}$ and 1,2-dichloropropane. Although the reproduction of the total data is not unreasonable, the convoluted $s$ wave peak is much more asymmetric than observed in the remaining compounds.

${ }^{1}$ K. Aflatooni and P. D. Burrow, J. Chem. Phys. 113, 1455 (2000).

${ }^{2}$ K. Aflatooni, G. A. Gallup, and P. D. Burrow, J. Phys. Chem. A 104, 7359 (2000)

${ }^{3}$ D. M. Pearl and P. D. Burrow, J. Chem. Phys. 101, 2940 (1994).

${ }^{4}$ K. Aflatooni and P. D. Burrow, Int. J. Mass. Spectrom. 205, 149 (2001).

${ }^{5}$ L. Sanche and G. J. Schulz, Phys. Rev. A 5, 1672 (1972).

${ }^{6}$ T. F. O’Malley, Phys. Rev. 150, 14 (1966).

${ }^{7}$ M. F. Falcetta and K. D. Jordan, J. Phys. Chem. 94, 5666 (1990).

${ }^{8}$ W. E. Wentworth, R. S. Becker, and R. Tung, J. Phys. Chem. 71, 1652 (1967). 
${ }^{9}$ I. I. Fabrikant and H. Hotop, Phys. Rev. A 63, 022706 (2001).

${ }^{10}$ H. A. Bethe, Phys. Rev. 47, 747 (1935);

${ }^{11}$ E. P. Wigner, Phys. Rev. 73, 1002 (1948).

${ }^{12}$ D. Klar, M.-W. Ruf, and H. Hotop, Int. J. Mass. Spectrom. 205, 93 (2001).

${ }^{13}$ J. N. Bardsley, A. Herzenberg, and F. Mandl, Atomic Collision Processes, edited by R. R. C. McDowell (North-Holland, Amsterdam, 1964), pp. 415-427. Also Proc. Phys. Soc. London 89, 321 (1966).

${ }^{14}$ Cited by T. F. O'Malley, Phys. Rev. 156, 230 (1967).

${ }^{15}$ W. Domcke, Phys. Rep. 208, 97 (1991).

${ }^{16}$ A. Stamatovic and G. J. Schulz, Rev. Sci. Instrum. 41, 423 (1970).

${ }^{17}$ S. C. Chu and P. D. Burrow, Chem. Phys. Lett. 172, 17 (1990).

${ }^{18}$ I. I. Fabrikant, Zh. Eksp. Teor. Fiz. 73, 1317 (1977) [Sov. Phys. JETP 46, 693 (1977)].

${ }^{19}$ P. D. Burrow, A. Modelli, N. S. Chiu, and K. D. Jordan, J. Chem. Phys. 77, 2699 (1982).

${ }^{20}$ M. Guerra, D. Jones, G. Distefano, F. Scagnolari, and A. Modelli, J. Chem. Phys. 94, 484 (1991).

${ }^{21}$ E. Vogt and G. H. Wannier, Phys. Rev. 95, 1190 (1954).

${ }^{22}$ Encyclopedia of Computational Chemistry, edited by P. V. Schleyer (Wiley, Chichester, UK, 1998), pp. 1439-1449.

${ }^{23}$ P. M. Morse, Phys. Rev. 34, 57 (1929).

${ }^{24}$ L. D. Landau and E. M. Lifshitz, Quantum Mechanics, 2nd ed. (Pergamon, New York, 1965), Sec. 90; I. S. Elets and A. K. Kazanskii, Sov. Phys. JETP 53, 499 (1981); see also, J. Heading, An Introduction to Phase-Integral Methods (Methuen, London, 1962).

${ }^{25}$ J. Franck, Trans. Faraday Soc. 21, 536 (1926).

${ }^{26}$ R. S. Wilde, G. A. Gallup, and I. I. Fabrikant, J. Phys. B 33, 5479 (2000).
${ }^{27}$ See, for example, Electron-Molecule Interactions and Their Applications, Vol. 1, edited by L. G. Christophorou (Academic, New York, 1984), p. 497.

${ }^{28}$ M. Abramowitz and I. Stegun, Handbook of Mathematical Functions, Applied Mathematics Series, Vol. 55 (NBS, Washington, D.C., 1964), Chap. 6.

${ }^{29}$ L. G. Christophorou, Z. Phys. Chem. (Leipzig) 195, 195 (1996).

${ }^{30}$ S. J. Burns, J. M. Matthews, and D. L. McFadden, J. Phys. Chem. 100, 19436 (1996).

${ }^{31}$ O. J. Orient, A. Chutjian, R. W. Crompton, and B. Cheung, Phys. Rev. A 39, 4494 (1989).

${ }^{32}$ D. Smith, N. G. Adams, and E. Alge, J. Phys. B 17, 461 (1984).

${ }^{33}$ P. Spanel, S. Matejcik and D. Smith, J. Phys. B 28, 2941 (1995).

${ }^{34}$ H. Shimamori, Y. Tatsuni, Y. Ogawa, and T. Sunagawa, J. Chem. Phys. 97, 6335 (1992).

${ }^{35}$ L. G. Christophorou, R. A. Mathis, D. R. James, and D. L. McCorkle, J. Phys. D 14, 1889 (1981).

${ }^{36}$ D. Smith, C. R. Herd, and N. G. Adams, Int. J. Mass Spectrom. Ion Processes 93, 15 (1989)

${ }^{37}$ R. P. Blaunstein and L. G. Christophorou, J. Chem. Phys. 49, 1526 (1968).

${ }^{38}$ E. Schultes, A. A. Christodoulides, and R. N. Schindler, Chem. Phys. 8, 354 (1975).

${ }^{39}$ R. W. Fessenden and K. M. Bansal, J. Chem. Phys. 53, 3468 (1970).

${ }^{40}$ T. Sunagawa and H. Shimamori, Int. J. Mass. Spectrom. 205, 285 (2001).

${ }^{41} \mathrm{~T}$. Wu and T. Ohmura, Quantum Theory of Scattering (Prentice-Hall, Englewood Cliffs, NJ, 1962), Sec. M3. 